\title{
Geometric construction of D-branes in WZW models
}

\section{G. Horcajada and F. Ruiz Ruiz}

Departamento de Física Teórica I, Universidad Complutense de Madrid, Ciudad Universitaria s/n, 28040 Madrid, Spain

E-mail: ghr@fis.ucm.es, ferruiz@fis.ucm.es

\begin{abstract}
The geometric description of D-branes in WZW models is pushed forward. Our starting point is a gluing condition $J_{+}=F J_{-}$that matches the model's chiral currents at the worldsheet boundary through a linear map $F$ acting on the WZW Lie algebra. The equivalence of boundary and gluing conditions of this type is studied in detail. The analysis involves a thorough discussion of Frobenius integrability, shows that $F$ must be an isometry, and applies to both metrically degenerate and nondegenerate D-branes. The isometry $F$ need not be a Lie algebra automorphism nor constantly defined over the brane. This approach, when applied to isometries of the form $F=R$ with $R$ a constant Lie algebra automorphism, validates metrically degenerate $R$-twined conjugacy classes as D-branes. It also shows that no D-branes exist in semisimple WZW models for constant $F=-R$.
\end{abstract}

Keywords: D-branes, Bosonic Strings

ARXIV EPRINT: 1104.4722 


\section{Contents}

1 Introduction 1

2 Gluing conditions for chiral currents $\quad 3$

3 Integration of the gluing condition and D-branes 5

3.1 Conditions for the existence of a D-brane 5

$\begin{array}{lll}3.2 & \text { Involutivity in detail } & 7\end{array}$

3.3 Reduction of isometric gluing conditions to boundary conditions 8

4 Limitations of the gluing condition approach 11

5 An application: D-branes from global isometries $\quad \mathbf{1 2}$

$\begin{array}{lll}5.1 & \text { Automorphisms and twined conjugacy classes as D-branes } & 13\end{array}$

$\begin{array}{lll}5.2 & \text { D-branes for semisimple Lie algebras } & 14\end{array}$

6 Some considerations on D-branes for local isometries 16

$\begin{array}{lll}7 & \text { Conclusion } & 17\end{array}$

$\begin{array}{ll}\text { A Alternative derivation of eq. (3.2) } & 18\end{array}$

$\begin{array}{ll}\text { B Metrically degenerate tangent planes } & 19\end{array}$

$\begin{array}{ll}\text { C Invariance of the spectrum of } \mathrm{Ad}_{g^{-1}} R & 20\end{array}$

\section{Introduction}

D-branes have become one of the main research topics in the string literature since the mid nineties. There are many reasons for this. Among them, the evidence that D-branes provide soliton and bound states in string backgrounds [1] and the realization that they become upon quantization noncommutative spacetimes [2-7].

Since WZW models are the building blocks of many string backgrounds [8-10], one sensible program to study D-branes and their properties is to consider their occurrence in models of this type. In fact, there are various approaches to D-branes in WZW models. Among them, the geometric approach [11-21], that regards D-branes as spacetime's submanifolds on which the string worldsheet boundary may be embedded, and the algebraic approach [22-29], that makes use of boundary conformal field theory.

In this paper we reexamine the geometric description of D-branes in a WZW model. The definition of D-brane that we will be using is the naïve geometric one; see section 2 
for details. A D p-brane in a string background $\left(G_{\mu \nu}, H_{\mu \nu \rho}\right)$ is any $(p+1)$-dimensional submanifold $N$ containing all possible motions for the string endpoints. These motions are specified by the boundary conditions for the string, which in turn can be viewed as a system of first order differential equations characterized by a two-form $\omega$ globally defined $^{1}$ on $N$ such that $d \omega=\left.H\right|_{N}$. A way to construct D-branes is thus to find all two-forms $\omega$ for which the boundary conditions can be integrated.

Our starting point for the geometric characterization of D-branes in a WZW model is a condition $J_{+}=F J_{-}$, called gluing condition, that matches the model's chiral currents $J_{-}$and $J_{+}$at the world sheet boundary through a linear map $F$ that acts on the model's Lie algebra. This matching condition is not a boundary condition, for it is not obtained by setting to zero the boundary term that arises from the variation of the model's classical action. However, it does specify, for every linear map $F$, vector fields characterizing tangent motions of the string endpoints. If these vector fields define an integrable distribution, they span the tangent bundle of a submanifold $N$ of the spacetime group manifold. The submanifold $N$ is a D-brane if the gluing condition can be written as a boundary condition with a two-form $\omega$ globally defined on $N$ such that $d \omega=\left.H\right|_{N}$.

We cross examine this approach for WZW models with arbitrary real Lie group G. Our only assumption is that the corresponding Lie algebra admits an invariant nondegenerate metric $\Omega$. This includes in particular noncompact group manifolds with Lorentzian signature, for which there exist metrically degenerate submanifolds $N$ such that the tangent space $T_{g} G$ at any point $g$ in $N$ cannot be written as an orthogonal sum $T_{g} N \oplus T_{g} N^{\perp}$.

If $F$ is a constant $\Omega$-preserving Lie algebra automorphism and the orthogonal decomposition $T_{g} G=T_{g} N \oplus T_{g} N^{\perp}$ is assumed, the vector fields defined by $F$ are known to be integrable and the two-form $\omega$ satisfying $d \omega=\left.H\right|_{N}$ is well known [11-14]. Our interest is in cases escaping these two assumptions. In this more general setting, the situation is very different. Firstly, because for an arbitrary linear map $F(g)$, the vector fields specified by the gluing condition, call them $t_{i}$, do not always define an integrable distribution. And secondly, because even if they do, the corresponding gluing condition cannot always be written as a boundary condition with a two-form $\omega$ globally defined on $N$ such that $d \omega=\left.H\right|_{N}$. In this regard, we prove the following two results. Every boundary condition for a D-brane $N$ can be written as a gluing condition $J_{+}=F J_{-}$, provided $\operatorname{det}\left(\left.G\right|_{N}-\omega\right) \neq 0$, where $\left.G\right|_{N}$ is the induced metric on $N$. And every gluing condition can be written as a boundary condition if the linear map $F(g)$ is an isometry of $\Omega$, in which case the two-form $\omega$ exists globally and is uniquely defined by its action $\omega\left(t_{i}, t_{j}\right)$ on the vector fields $t_{i}$ defined by $F(g)$. For a general isometry $F(g)$, the requirement $d \omega=\left.H\right|_{N}$ however does not hold but it becomes a matter of straightforward algebra to check it in every instance. These two results open some problems, among them studying the applicability of this approach to D-branes for which a full set of gluing conditions is not known [19, 35-37].

The paper is organized as follows. Section 2 poses the problem and reviews the description of D-branes in WZW models in terms of the gluing condition for the chiral currents.

\footnotetext{
${ }^{1}$ We will use $B$ for two-forms locally defined on the whole group manifold such that $d B=H$, and the Greek letter $\omega$ for the two-form globally defined on the submanifold $N$.
} 
In section 3, integrability in terms of Frobenius theorem is studied and it is shown that the two-form $\omega$ for which the gluing condition becomes a boundary condition exists if and only if $F(g)$ is an isometry of $\Omega$. Section 4 contains a discussion of the limitations of the gluing condition approach. Isometries of the form $F= \pm R$, where $R$ is a constant Lie algebra automorphism, are considered in section 5 . The case $F=R$ has been studied by other Authors [11-14] under the hypothesis that $T_{g} G=T_{g} N \oplus T_{g} N^{\perp}$, the resulting D-branes being $R$-twined conjugacy classes. It is shown that this result holds even if the latter assumption on $T_{g} G$ fails. As regards the case $F=-R$, it is proved that, contrarily to some claims, the gluing condition for $F=-R$ does not provide D-branes for semisimple Lie algebras. In section 6, some examples of $g$-dependent isometries $F(g)$ are considered. It is shown that two different isometries may define the same integrable distribution but different two-forms $\omega$, one of them satisfying $d \omega=\left.H\right|_{N}$, hence defining a D-brane, and the other one not. We close the paper with our conclusions and three short appendices collecting technical points.

\section{Gluing conditions for chiral currents}

In the sigma model approach, a D $p$-brane in a string background $\left(G_{\mu \nu}, H_{\mu \nu \lambda}\right)$ is a $(p+1)$ dimensional submanifold $N$ on which the endpoints of an open string may lie. The submanifold $N$ has embedded coordinates $x^{\mu}(\tau)=\left.X^{\mu}(\tau, \sigma)\right|_{\partial \Sigma}$ and these must satisfy the boundary conditions

$$
\left.\left(\partial_{i} f^{\mu} G_{\mu \nu} \partial_{\sigma} X^{\nu}-\omega_{i j} \partial_{\tau} \alpha^{j}\right)\right|_{\partial \Sigma}=0 \quad i=1, \ldots, p+1 .
$$

Here $\alpha^{1}, \ldots, \alpha^{p+1}$ are local coordinates on the $\mathrm{D} p$-brane, so that $x^{\mu}=f^{\mu}\left(\alpha^{1}, \ldots, \alpha^{p+1}\right)$, and $\omega_{i j}$ are the components of a two-form $\omega$ globally defined on the brane such that $d \omega=\left.H\right|_{N}$.

We are interested in D-branes in string backgrounds described by WZW models [30] with real Lie group $G$ and Lie algebra $\mathfrak{g}$, both of dimension d. The Lie algebra $\mathfrak{g}$ is a vector space over $\mathbf{R}$ and has generators $\left\{T_{A}\right\}$ with commutation relations

$$
\left[T_{A}, T_{B}\right]=f_{A B}^{C} T_{C} \quad A, B, C=1, \ldots, \mathrm{d} .
$$

The algebra $\mathfrak{g}$ is assumed to have a nondegenerate invariant metric $\Omega$, of arbitrary signature, with components $\Omega_{A B}=\Omega\left(T_{A}, T_{B}\right)$, so that

$$
\Omega\left(\left[T_{A}, T_{B}\right], T_{C}\right)=\Omega\left(T_{A},\left[T_{B}, T_{C}\right]\right) \Leftrightarrow f_{A B}{ }^{D} \Omega_{D C}=\Omega_{A D} f_{B C} D .
$$

The existence of such a metric is the only restriction on $\mathfrak{g}$. The group $G$ is taken as the connected component obtained from $\mathfrak{g}$ through exponentiation.

If $X^{\mu}$ are local coordinates in $G$, the left-invariant $e^{A}{ }_{\mu}$ and right-invariant $\bar{e}^{A}{ }_{\mu}$ vielbeins that map the group $G$ to its tangent space $T_{g} G$ at $g$ are defined by

$$
g^{-1} d g=T_{A} e_{\mu}^{A} d X^{\mu} \quad d g g^{-1}=T_{A} \bar{e}_{\mu}^{A} d X^{\mu} .
$$

In terms of them, the adjoint action of the group on the Lie algebra is

$$
\operatorname{Ad}_{g}\left(T_{A}\right)=g T_{A} g^{-1}=T_{B} \bar{e}^{B}{ }_{\mu}\left(e^{-1}\right)^{\mu} A \quad \Leftrightarrow \quad \operatorname{Ad}_{g}=\bar{e} e^{-1} .
$$


The string background $\left(G_{\mu \nu}, H_{\mu \nu \lambda}\right)$ is defined from $\Omega$ by

$$
\begin{aligned}
G_{\mu \nu} & =\Omega\left(g^{-1} \partial_{\mu} g, g^{-1} \partial_{\nu} g\right) \\
H_{\mu \nu \lambda} & =\Omega\left(\left[g^{-1} \partial_{\mu} g, g^{-1} \partial_{\nu} g\right], g^{-1} \partial_{\lambda} g\right) .
\end{aligned}
$$

By construction, the metric $G_{\mu \nu}$ is bi-invariant,

$$
G_{\mu \nu}=e_{\mu}^{A} \Omega_{A B} e^{B}{ }_{\nu}=\bar{e}^{A}{ }_{\mu} \Omega_{A B} \bar{e}^{B}{ }_{\nu} \Leftrightarrow G=e^{\mathrm{T}} \Omega e=\bar{e}^{\mathrm{T}} \Omega \bar{e},
$$

the superscript $\mathrm{T}$ denoting transposition. In this paper the standard notation $G(a, b)=$ $G_{\mu \nu} a^{\mu} b^{\nu}$ will be used.

The WZW classical action for the open string in the background $\left(G_{\mu \nu}, H_{\mu \nu \lambda}\right)$ can be written as [31]

$$
S_{\mathrm{WZW}}=\frac{k}{4 \pi} \int_{\Sigma} d^{2} \sigma \Omega\left(g^{-1} \partial_{a} g, g^{-1} \partial^{a} g\right)+\frac{k}{4 \pi}\left(\int_{\Sigma} g^{*} B+\int_{\partial \Sigma} g^{*} A\right),
$$

with $g=g\left(X^{\mu}(\tau, \sigma)\right)$ and $\sigma^{a}=(\tau, \sigma)$ world sheet indices. Here $B$ is any two-form defined on $G$ such that $H=d B$, and $g^{*} B$ is its pullback. The form $B$ may not be globally defined, but must exist locally. This is the case, for example, if $H$ is not exact. The one-form $A$ is defined on the D-brane, exists at least locally and is such that $d A=\omega-\left.B\right|_{N}$. See ref. [31] for details.

In worldsheet coordinates $\sigma^{ \pm}=\tau \pm \sigma$, the field equations read $\partial_{+} J_{-}=\partial_{-} J_{+}=0$, where the chiral currents $J_{-}$and $J_{+}$are given by

$$
J_{-}\left(\sigma^{-}\right)=g^{-1} \partial_{-} g \quad J_{+}\left(\sigma^{+}\right)=-\partial_{+} g g^{-1} .
$$

Due to the simplicity of the solutions for $J_{+}$and $J_{-}$, we are interested in formulating the boundary conditions for a D-brane in terms of $J_{+}$and $J_{-}$. We will then assume that there exists a mapping $F$ from $\mathfrak{g}$ to $\mathfrak{g}$ relating the two currents at the world sheet boundary, that is, $J_{+}=F\left(J_{-}\right)$at $\sigma^{+}=\sigma^{-}$. Recalling that D-branes in the sigma model approach are defined by boundary conditions of order one in $\left.\partial_{ \pm} X^{\mu}\right|_{\partial \Sigma}$ and noting that $J_{-}$and $J_{+}$are already order one in $\partial_{ \pm} X^{\mu}$, we restrict ourselves to linear maps $F(g)$ that may depend on $g$ but not on $J_{ \pm}(g)$. For the chiral currents at a D-brane we thus require

$$
\left.J_{+}\right|_{\sigma^{+}=\sigma^{-}}=\left.F(g) J_{-}\right|_{\sigma^{+}=\sigma^{-}},
$$

with $F(g)$, for every $g$ in $N$, a linear map that acts on $\mathfrak{g}$ as a vector space. The linear map $F(g)$ is represented by a real $\mathrm{d} \times \mathrm{d}$ matrix with entries $F_{B}^{A}$ given by $F(g) T_{B}=T_{A} F^{A}{ }_{B}(g)$. It is important to note that eq. (2.8) is not a boundary condition derived from the classical action above but a working hypothesis formulated ad hoc. To keep this in mind, eq. (2.8) is called gluing condition, rather than boundary condition. We will take it as starting point for the construction of D-branes.

Eq. (2.8) defines a D-brane if it can be written as a sigma model boundary condition (2.1), with $\omega$ a two-form globally defined on $N$ such that [31]

$$
\left.H\right|_{N}=d \omega .
$$


We will show in section 3 that every boundary condition (2.1) can be written as a gluing condition (2.8), with $F$ and isometry of $\Omega$, except for D-branes $N$ such that $\operatorname{det}\left(\left.G\right|_{N}-\omega\right)=0$, where $\left.G\right|_{N}$ is the induced metric on $N$. Apart from these instances, the gluing condition is capable of constructing all D-branes defined by boundary conditions.

It is convenient to write the gluing condition (2.8) in terms of local coordinates $X^{\mu}$. To do this [13], it is enough to use for the chiral currents their expressions

$$
J_{-}=\partial_{-} X^{\mu} e^{A}{ }_{\mu} T_{A} \quad J_{+}=-\partial_{+} X^{\mu} \bar{e}_{\mu}^{A} T_{A}
$$

in terms of the string coordinates $X^{\mu}$ and the left and right-invariant vielbeins $e^{A}{ }_{\mu}$ and $\bar{e}_{\mu}^{A}$. This yields

$$
\left.\partial_{+} X^{\mu}\right|_{\partial \Sigma}=\left.\mathcal{F}_{\nu}^{\mu} \partial_{-} X^{\nu}\right|_{\partial \Sigma},
$$

where the matrix $\mathcal{F}$ is defined by

$$
\mathcal{F}(x)=-\bar{e}^{-1} F(g) e .
$$

In worldsheet coordinates $\tau$ and $\sigma$, eq. (2.10) takes the form

$$
\left.(\mathcal{F}-1) \partial_{\tau} X\right|_{\partial \Sigma}=\left.(\mathcal{F}+1) \partial_{\sigma} X\right|_{\partial \Sigma}
$$

We emphasize that the matrix $\mathcal{F}(x)$ is defined on $N$ and depends on the string endpoints coordinates $x^{\mu}=\left.X^{\mu}\right|_{\partial \Sigma}$ through $e(x), \bar{e}(x)$ and $F(g(x))$. It however acts on arbitrary tangent vectors in $T_{g} G$. To ease the notation, whenever there is no confusion we will remove from $F(g)$ and $\mathcal{F}(g)$ the dependence on $g$.

\section{$3 \quad$ Integration of the gluing condition and D-branes}

In this section, the gluing condition (2.12) is explicitly solved for $\left.\partial_{\tau} X^{\mu}\right|_{\partial \Sigma}$. The solution happens to be given in terms of vector fields defined by the linear map $F$. The involutivity requisite that such fields must satisfy to define a foliation of $G$ in terms of a family of submanifolds $N$ is studied in detail. Finally, it is shown that the gluing condition for a linear map $F$ takes the form of a boundary condition if and only if $F$ is an isometry of $\Omega$, and the two-form $\omega$ is constructed from $F$. When the resulting $\omega$ satisfies $d \omega=\left.H\right|_{N}$, the submanifold $N$ is a D-brane.

\subsection{Conditions for the existence of a D-brane}

The set of possible motions of the string endpoints at an arbitrary spacetime point $g$ is the set $\Pi_{g}$ of solutions $t^{\mu}(x):=\left.\partial_{\tau} X^{\mu}\right|_{\partial \Sigma}$ to the gluing condition (2.12) for some $u^{\mu}(x):=\left.\partial_{\sigma} X^{\mu}\right|_{\partial \Sigma}$. Since $t(x)=t^{\mu}(x) \partial_{\mu}$ and $u(x)=u^{\mu}(x) \partial_{\mu}$ are tangent vectors to $G$ at $g$, we may write

$$
\Pi_{g}=\left\{t \in T_{g}(G):[\mathcal{F}(g)-1] t=[\mathcal{F}(g)+1] u \text { for } u \in T_{g}(G)\right\} .
$$

Equivalently,

$$
\Pi_{g}=\left\{t \in T_{g}(G):(\mathcal{F}-1) t \in \operatorname{Im}(\mathcal{F}+1)\right\}
$$


The set $\Pi_{g}$ is a linear subspace of the tangent space $T_{g} G$ and we will often call it the tangent plane at $g$ defined by $F$.

Consider $v \in \operatorname{Im}(\mathcal{F}+1)$, so that there exists $w$ in $T_{g}(G)$ such that $v=(\mathcal{F}+1) w$. It follows that $(\mathcal{F}-1) v=(\mathcal{F}+1)(\mathcal{F}-1) w$ belongs to $\operatorname{Im}(\mathcal{F}+1)$. Hence $v$ is in $\Pi_{g}$ and

$$
\operatorname{Im}(\mathcal{F}+1) \subset \Pi_{g}
$$

Consider now $v^{\prime}$ in $\Pi_{g}$. It then exists $w^{\prime}$ in $T_{g} G$ such that $(\mathcal{F}-1) v^{\prime}=(\mathcal{F}+1) w^{\prime}$. This implies that $v^{\prime}=\frac{1}{2}(\mathcal{F}+1)\left(v^{\prime}-w^{\prime}\right)$, so that $v^{\prime}$ belongs to $\operatorname{Im}(\mathcal{F}+1)$ and

$$
\Pi_{g} \subset \operatorname{Im}(\mathcal{F}+1)
$$

Hence

$$
\Pi_{g}=\operatorname{Im}(\mathcal{F}+1) .
$$

An alternative derivation of this result in terms of the eigenvectors of $\mathcal{F}$ can be found in appendix A.

Since the gluing condition (2.12) holds for arbitrary $g$, the solution $t(x)$ defines a vector field for a given $u(x)$. If $M$ is a submanifold of $G$, we define

$$
\Pi^{M}=\left\{\left(g, \Pi_{g}\right): g \in M\right\}
$$

$\Pi^{M}$ is a distribution on $M$ if the tangent plane $\Pi_{g}$ has the same dimension for all $g$ in $M$. According to Frobenius theorem [33], a distribution $\Pi^{M}$ is integrable if and only if it is involutive. Integrability ensures that $\Pi_{g}$ is, for all $g$ in $M$, not just a tangent plane but the tangent space to a submanifold $N$ of $M$, that is $\Pi_{g}=T_{g} N$. Involutivity states that the commutator of any two vector fields $t_{1}$ and $t_{2}$ taking values in $\Pi^{M}$ also takes values in $\Pi^{M}$,

$$
\left[t_{1}, t_{2}\right](g) \in \Pi_{g}
$$

For the manifold $N$ to define a D-brane, it must contain all the points $g$ in $G$ connected by the integral curves of the vector fields $t$. This condition cannot be relaxed, since one would then leave out from the D-brane points at which the open string may end. See section 4 for examples.

As a practical matter, to determine if a linear map $F$ defines a D-brane, one may proceed in three steps:

Step 1. Study the rank of the matrix $\mathcal{F}(g)+1$ as a function of $g$. Consider a submanifold $D_{n}(F)$ formed by the points $g$ in $G$ such that (i) the rank of $\mathcal{F}(g)+1$ is $n$, and (ii) $g$ is not connected by integral curves of the vector fields $t$ with points $g^{\prime}$ at which the rank of $\mathcal{F}\left(g^{\prime}\right)+1$ is different from $n$.

Step 2. Check the involutivity condition (3.4) in $D_{n}(F)$. If it holds, the distribution $\Pi^{D_{n}(F)}$ is the tangent bundle of a submanifold $N$ of $G$ of dimension $n$, or more precisely of a family of submanifolds which foliate $D_{n}(F)$. 
Step 3. Find a two-form $\omega$ globally defined on $N$ for which the gluing condition for $F$ can be recast as a sigma model boundary condition and such that $d \omega=\left.H\right|_{N}$. If such a $\omega$ exists, the submanifold $N$ is a D-brane of dimension $n$.

In what follows we further elaborate these three steps.

\subsection{Involutivity in detail}

The definition of $\mathcal{F}$ in (2.11) and the expression for the group adjoint action in (2.4) imply that $\mathcal{F}+1=e^{-1}\left(-\operatorname{Ad}_{g^{-1}} F+1\right) e$. The space of tangent directions $\Pi_{g}=\operatorname{Im}(\mathcal{F}+1)$ can then be written as

$$
\Pi_{g}=g\left[\operatorname{Ad}_{g^{-1}} F(g)-1\right] \mathfrak{g} .
$$

For every $V$ in the Lie algebra $\mathfrak{g}$,

$$
g\left[\operatorname{Ad}_{g^{-1}} F(g)-1\right] V=F(g) V g-g V
$$

is a vector field. It is actually the sum of a right-invariant vector field $Y g$, with $Y=F(g) V$, and a left-invariant vector field $g Y$, with $Y=-V$.

Right and left-invariant vector fields act on differentiable functions $f$ defined on $G$ and taking values in $\mathbf{R}$ according to

$$
Y g(f(g))=\left.\frac{d}{d t} f\left(e^{t Y} g\right)\right|_{t=0} \quad g Y(f(g))=\left.\frac{d}{d t} f\left(g e^{t Y}\right)\right|_{t=0} .
$$

If $g$ is parameterized by coordinates $x^{\mu}$, the vector field components of $Y g$ and $g Y$ are

$$
\begin{aligned}
& Y g=Y^{A} T_{A} g=Y^{A}\left(\bar{e}^{-1}\right)_{A}^{\mu} \partial_{\mu}=(Y g)^{\mu} \partial_{\mu} \\
& g Y=Y^{A} g T_{A}=Y^{A}\left(e^{-1}\right)^{\mu}{ }_{A} \partial_{\mu}=(g Y)^{\mu} \partial_{\mu} .
\end{aligned}
$$

These equations and $V^{A}=e_{\mu}^{A} v^{\mu}$ provide $F(g) V g-g V=-[(\mathcal{F}+1) v]^{\mu} \partial_{\mu}$, which again gives for the vector field $t$ the form used in eq. (3.2). Since $\left\{T_{A}\right\}$ is a basis of $\mathfrak{g}$, the vector fields that define $\Pi_{g}$ read

$$
t_{A}=F T_{A} g-g T_{A}=\left[\left(\bar{e}^{-1}\right)_{B}^{\mu} F_{A}^{B}-\left(e^{-1}\right)_{A}^{\mu}\right] \partial_{\mu} .
$$

These fields completely determine the motions of the string endpoints solving the gluing condition with linear map $F$.

The rank of $\mathcal{F}+1$ is obviously equal to the rank of $\operatorname{Ad}_{g^{-1}} F(g)-1$. Say it takes the value $n$ for all $g$ in a domain $D_{n}(F)$ in $G$. Assume further that the integral curves of the fields $t_{A}$ are in $D_{n}(F)$. The involutivity condition (3.4) requires that, for all $U$ and $V$ in $\mathfrak{g}$ and for all $g$ in $D_{n}(F)$, there exist $W$ in $\mathfrak{g}$ such that

$$
[F(g) U g-g U, F(g) V g-g V]=F(g) W g-g W .
$$

It is important to keep in mind that $W$ need not be the same for all $g$. Eq. (3.9) is an equation in $F(g)$, in the sense that $W$ does not exist for every linear map $F(g)$. After expanding its left hand side, it becomes

$$
[F(g) U g, F(g) V g]-[F(g) U g, g V]-[g U, F(g) V g]+[g U, g V]=F(g) W g-g W .
$$


Let us understand each one of the terms in this expression. Using eqs. (3.7), the action of first term on an arbitrary function $f$ is

$$
[F(g) U g, F(g) V g](f(g))=\left.\frac{\partial^{2}}{\partial s \partial t} f\left(e^{t F\left(e^{s F(g) U} g\right) V} e^{s F(g) U} g\right)\right|_{s=t=0}-(U \leftrightarrow V) .
$$

After performing the derivatives with respect to $s$ and $t$ and using eqs. (3.7), this reduces to

$$
\begin{aligned}
{[F(g) U g,} & F(g) V g](f(g))=[F(g) V, F(g) U] g(f(g)) \\
& +(F(g) U g(F(g))) V g(f(g))-(F(g) V g(F(g))) U g(f(g))
\end{aligned}
$$

Proceeding similarly for the other commutators in eq. (3.10), we obtain

$$
[F(g) U g, g V](f(g))=-(g V(F(g))) U g(f(g))
$$

and

$$
[g U, g V](f(g))=g[U, V](f(g)) .
$$

Taking eqs. (3.11)-(3.13) to eq. (3.10) and noting that $f$ is arbitrary, we finally arrive at

$$
\begin{aligned}
-[F U, F V] g & +g[U, V]=F W g-g W \\
& -((F U g-g U)(F)) V g+((F V g-g V)(F)) U g .
\end{aligned}
$$

The last two terms in the right hand side carry the action of the vector fields $F(g) U g-g U$ and $F(g) V g-g V$ on $F(g)$ as a function of $g$, the result being two linear operators that act on $V$ and $U$.

If $F$ does not depend on $g$, the action of $F U g-g U$ and $F V g-g V$ on $F$ is zero and eq. (3.14) simplifies to

$$
-[F U, F V] g+g[U, V]=F W g-g W .
$$

\subsection{Reduction of isometric gluing conditions to boundary conditions}

Assume that the linear map $F(g)$ is such that steps 1 and 2 are satisfied. There is then a submanifold $N$ whose tangent bundle $\Pi^{N}$ is formed by the tangent spaces $T_{g} N=\operatorname{Im}(\mathcal{F}+1)$ for all $g$ in $N$. In what follows we show that the necessary and sufficient condition for the gluing condition (2.12) to be equivalent to a boundary condition (2.1) is that $F(g)$ is an isometry of the Lie algebra metric $\Omega$.

Since $\partial_{\tau} x$ belongs to $T_{g} N$, there exists $v$ in $T_{g} G$ such that $\partial_{\tau} x=(\mathcal{F}+1) v$ and the boundary condition (2.1) can be recast as

$$
G\left(u_{0},\left.\partial_{\sigma} X\right|_{\partial \Sigma}\right)=\omega\left(u_{0},(\mathcal{F}+1) v\right) \quad \text { for all } \quad u_{0} \in \operatorname{Im}(\mathcal{F}+1)
$$

The gluing condition (2.12) can in turn be written as

$$
\left.(\mathcal{F}+1) \partial_{\sigma} X\right|_{\partial \Sigma}=(\mathcal{F}-1)(\mathcal{F}+1) v
$$


This can be viewed as an equation in $\left.\partial_{\sigma} X\right|_{\partial \Sigma}$, whose solutions are of the form

$$
\left.\partial_{\sigma} X\right|_{\partial \Sigma}=(\mathcal{F}-1) v+v_{0}
$$

with arbitrary $v_{0}$ in $\operatorname{Ker}(\mathcal{F}+1)$. Eq. (3.17) implies that

$$
G\left(u_{0},\left.\partial_{\sigma} X\right|_{\partial \Sigma}\right)=G\left(u_{0},(\mathcal{F}-1) v\right)+G\left(u_{0}, v_{0}\right) \text { for all } u_{0} \in \operatorname{Im}(\mathcal{F}+1) .
$$

Of the two terms on the right hand side, only the first one is linear in $v$. From this and the linearity in $v$ of the boundary condition (3.16), we conclude that eq. (3.18) is compatible with the boundary condition (3.16) if and only if the following two requisites are met:

(1) $G\left(u_{0}, v_{0}\right)=0$ for all $u_{0}$ in $\operatorname{Im}(\mathcal{F}+1)$ and all $v_{0}$ in $\operatorname{Ker}(\mathcal{F}+1)$, and

(2) the action of the two-form $\omega$ on arbitrary $(\mathcal{F}+1) u$ and $(\mathcal{F}+1) v$ in $T_{g} N$ is given by

$$
\omega((\mathcal{F}+1) u,(\mathcal{F}+1) v)=G((\mathcal{F}+1) u,(\mathcal{F}-1) v) .
$$

For eq. (3.19) to make sense, its right hand side must be antisymmetric,

$$
0=G((\mathcal{F}+1) u,(\mathcal{F}-1) v)+G((\mathcal{F}+1) v,(\mathcal{F}-1) u)=2 G(\mathcal{F} u, \mathcal{F} v)-2 G(u, v) .
$$

Since $u$ and $v$ are arbitrary in $T_{g} G$, the operator $\mathcal{F}$, defined on $N$, acts isometrically on the whole tangent space $T_{g} G$,

$$
G(\mathcal{F} u, \mathcal{F} v)=G(u, v)
$$

This in turn implies that

$$
\operatorname{Im}(\mathcal{F} \pm 1)=\operatorname{Ker}(\mathcal{F} \pm 1)^{\perp}
$$

and makes condition (1) trivial. Furthermore, given $v$ in $T_{g} G$, consider $v^{\prime}=v+v_{0}^{\prime}$ in $T_{g} G$, with arbitrary $v_{0}^{\prime}$ in $\operatorname{Ker}(\mathcal{F}+1)$. From eq. (3.21) it follows that

$$
G\left((\mathcal{F}+1) u,(\mathcal{F}-1) v^{\prime}\right)=G((\mathcal{F}+1) u,(\mathcal{F}-1) v) .
$$

In other words, the right hand side in eq. (3.19) does not depend on the choice of $v_{0}$ in (3.17) and the two-form $\omega$ as defined by eq. (3.19) is single valued. Finally, $\omega$ exists globally on $N$ since it is given by eq. (3.19) through its action on arbitrary vectors $(\mathcal{F}+1) u$ and $(\mathcal{F}+1) v$ in $T_{g} N$ for any $g$ in $N$.

From the definition (2.11) of $\mathcal{F}$, the bi-invariance property (2.6) of the metric $G$ and eq. (3.20), it is straightforward that

$$
\Omega\left(F(g) T_{A}, F(g) T_{B}\right)=\Omega\left(T_{A}, T_{B}\right)
$$

for all $T_{A}$ and $T_{B}$ in the Lie algebra. This shows that the linear map $F(g)$ is an isometry of the Lie algebra metric $\Omega$.

Note that if $\mathcal{F}$ is an isometry on $T_{g} G$, eq. (3.18) not only follows from the gluing condition (2.12) but is equivalent to it. All in all we have that the necessary and sufficient 
condition for the gluing condition to have the form of a boundary condition is that $F(g)$ is an isometry of $\Omega$, the two-form $\omega$ being given by eq. (3.19). In what follows whenever we write $F(g)$ we will be thinking of it as an isometry. In terms of the fields $t_{A}=g\left[\operatorname{Ad}_{g^{-1}} F(g)-1\right] T_{A}$ in eq. (3.8), the definition of $\omega$ in eq. (3.19) can be written as

$$
\omega\left(t_{A}, t_{B}\right)=\Omega\left(\left(\operatorname{Ad}_{g^{-1}} F-1\right) T_{A},\left(\operatorname{Ad}_{g^{-1}} F+1\right) T_{B}\right) .
$$

We remark that the analysis performed here holds for any linear map $F$, regardless of whether it is constant or $g$-dependent.

The condition that $\omega$ must satisfy for $N$ to be a D-brane is

$$
d \omega=\left.H\right|_{N},
$$

where the exterior derivative on the left hand side is taken with respect to the directions in $T_{g} N$ and not with respect to arbitrary directions in $T_{g} G$. Condition (3.24) does not hold for every isometry $F$ defining a submanifold $N$ upon integration of the gluing condition. Examples of this are given in section 6 and in ref. [21]. Let us discuss some cases in which $\omega$ fulfills eq. (3.24).

For one and two-dimensional submanifolds $N$, eq. (3.24) trivially holds and the only requirement for the existence of a D-brane for an isometry $F$ is involutivity. Assume now that $N$ has dimension larger than two and that $F=R$ is a $g$-independent, $\Omega$-preserving Lie algebra automorphism. The exterior derivative of $\omega$ on $N$ is a three-form whose action on vector fields $t_{1}, t_{2}$ and $t_{3}$ in $T_{g} N$ is given by

$$
\begin{aligned}
d \omega\left(t_{1}, t_{2}, t_{3}\right)= & t_{1}\left(\omega\left(t_{2}, t_{3}\right)\right)-\omega\left(\left[t_{1}, t_{2}\right], t_{3}\right) \\
& +t_{2}\left(\omega\left(t_{3}, t_{1}\right)\right)-\omega\left(\left[t_{2}, t_{3}\right], t_{1}\right) \\
& +t_{3}\left(\omega\left(t_{1}, t_{2}\right)\right)-\omega\left(\left[t_{3}, t_{1}\right], t_{2}\right) .
\end{aligned}
$$

Since the vector fields $t_{A}=g\left(\operatorname{Ad}_{g^{-1}} R-1\right) T_{A}$ span $T_{g} N$, it suffices to calculate $d \omega\left(t_{A}, t_{B}, t_{C}\right)$. For that, we need to consider terms of the form $t_{A}\left(\omega\left(t_{B}, t_{C}\right)\right)$ and $\omega\left(\left[t_{A}, t_{B}\right], t_{C}\right)$. Since $R$ does not depend on $g$,

$$
t_{A}\left(\omega\left(t_{B}, t_{C}\right)\right)=\Omega\left(t_{A}\left(\operatorname{Ad}_{g^{-1}}\right) R T_{B}, T_{C}\right)-\Omega\left(t_{A}\left(\operatorname{Ad}_{g^{-1}}\right) R T_{C}, T_{B}\right) .
$$

Noting that

$$
t_{A}\left(\operatorname{Ad}_{g^{-1}}\right) V=-\left[\left(\operatorname{Ad}_{g^{-1}} R-1\right) T_{A}, \operatorname{Ad}_{g^{-1}} V\right]
$$

for all $V$ in $\mathfrak{g}$, using that $R$ and $\operatorname{Ad}_{g^{-1}} R$ are Lie algebra automorphisms and recalling that $\Omega$ is invariant, it is straightforward to see that

$$
t_{A}\left(\omega\left(t_{B}, t_{C}\right)\right)=\Omega\left(\operatorname{Ad}_{g^{-1}} R T_{B},\left[T_{C}, T_{A}\right]\right)-\Omega\left(T_{B}, \operatorname{Ad}_{g^{-1}} R\left[T_{C}, T_{A}\right]\right)-(B \leftrightarrow C) .
$$

Proceeding similarly with $\omega\left(\left[t_{A}, t_{B}\right], t_{C}\right)$, one has

$$
\omega\left(\left[t_{A}, t_{B}\right], t_{C}\right)=\Omega\left(\left[T_{A}, T_{B}\right], \operatorname{Ad}_{g^{-1}} R T_{C}\right)-\Omega\left(\operatorname{Ad}_{g^{-1}} R\left[T_{A}, T_{B}\right], T_{C}\right) .
$$


Upon substitution in eq. (3.25), this gives

$$
d \omega\left(t_{A}, t_{B}, t_{C}\right)=\Omega\left(\left[\left(\operatorname{Ad}_{g^{-1}} R-1\right) T_{A},\left(\operatorname{Ad}_{g^{-1}} R-1\right) T_{B}\right],\left(\operatorname{Ad}_{g^{-1}} R-1\right) T_{C}\right) .
$$

On the other hand, from eq. (2.5) it trivially follows that the right hand side in (3.26) is equal to $H\left(t_{A}, t_{B}, t_{C}\right)$. Hence, for any constant isometry that is also a Lie algebra automorphism, $d \omega=\left.H\right|_{N}$. It is clear that if $F$ depends on $g$ and/or is not a Lie algebra automorphism, this proof does not stand. In these cases, condition (3.24) can always be checked by hand. See ref. [21] for examples.

We end this section by remarking that we have not assumed at any stage that $T_{g} G=\Pi_{g} \oplus \Pi_{g}^{\perp}$, thus generalizing previous approaches [14] that, under such an assumption, define $\omega$ for $F$ a constant Lie algebra automorphism. In this regard, it is worth noting that $T_{g} G=\Pi_{g} \oplus \Pi_{g}^{\perp}$ holds for Lie groups with Euclidean signature metric $G_{\mu \nu}$. However, if $G_{\mu \nu}$ is Lorentzian, it may occur that, among the vector fields defining the distribution $\Pi^{M}$, one of them is null and orthogonal to all the others. If this is the case, the induced metric on the D-brane is degenerate and the tangent space $T_{g} G$ cannot be written as a direct sum of $\Pi_{g}$ and $\Pi_{g}^{\perp}$. In appendix B an explicit construction of such null vector fields in terms of the eigenvectors of $\mathcal{F}$ is presented, and in ref. [21] a family of degenerate D2-branes for the Nappi-Witten [34] model is found.

\section{Limitations of the gluing condition approach}

In the previous section we have shown that every gluing condition with $F$ an isometry takes the form of a boundary condition with a two-form $\omega$ defined by eq. (3.19). It may, however, occur that a boundary condition describing a D-brane cannot be written as a gluing condition. In this section we tackle this problem and show that every boundary condition with two-form $\omega$ defining a D-brane $N$ can be written as a gluing condition if and only if $\operatorname{det}\left(\left.G\right|_{N}-\omega\right) \neq 0$.

Let us then consider a D-brane $N$ with tangent space $T_{g} N$ specified by the boundary condition (2.1), the two-form $\omega$ acting on $T_{g} N$. It is most convenient for our purposes to write the boundary condition as

$$
G\left(\delta X,\left.\partial_{\sigma} X\right|_{\partial \Sigma}\right)=\omega\left(\delta X,\left.\partial_{\tau} X\right|_{\partial \Sigma}\right) \quad \text { for all } \delta X \in T_{g} N
$$

with $\left.\partial_{\sigma} X\right|_{\partial \Sigma}$ in $T_{g} G$ and $\left.\partial_{\tau} X\right|_{\partial \Sigma}$ in $T_{g} N$. We now define a map $\mathcal{K}: T_{g} N \rightarrow T_{g} G /\left(T_{g} N^{\perp}\right)$ whose action on $w$ in $T_{g} N$ is given by

$$
G(z, \mathcal{K} w)=\frac{1}{2}[G(z, w)-\omega(z, w)] \text { for all } \quad z \in T_{g} N .
$$

The map $\mathcal{K}$ is trivially linear and takes values in the quotient $T_{g} G /\left(T_{g} N^{\perp}\right)$. To see the latter, assume that $y$ in $T_{g} G$ is such that $G(z, y)=G(z, \mathcal{K} w)$ for all $z$ in $T_{g} N$. It follows that $G(z, y-\mathcal{K} w)=0$, which in turn implies that $y-\mathcal{K} w$ is in $T_{g} N^{\perp}$.

Furthermore, $\mathcal{K}$ is injective if and only if $\operatorname{det}\left(\left.G\right|_{N}-\omega\right) \neq 0$. Indeed, for $w^{\prime} \neq w$ in $T_{g} N$ such that $\mathcal{K} w^{\prime}=\mathcal{K} w$, we have, according to eq. (4.2), that

$$
G\left(z, w^{\prime}-w\right)=\omega\left(z, w^{\prime}-w\right) \quad \text { for all } \quad z \in T_{g} N .
$$


A vector $w^{\prime}-w \neq 0$ satisfying this condition exists if and only if $\operatorname{det}\left(\left.G\right|_{N}-\omega\right)=0$, which proves the statement. Actually, since $\operatorname{dim}\left(T_{g} N\right)+\operatorname{dim}\left(T_{g} N^{\perp}\right)=\operatorname{dim}\left(T_{g} G\right)$, the map $\mathcal{K}$ is bijective if it is injective.

For $\operatorname{det}\left(\left.G\right|_{N}-\omega\right) \neq 0$, the inverse map $\mathcal{K}^{-1}: T_{g} G /\left(T_{g} N^{\perp}\right) \rightarrow T_{g} N$ hence exists and is bijective. From $\mathcal{K}^{-1}$ we define a linear map $\mathcal{G}: T_{g} G \rightarrow T_{g} N$ whose action on an arbitrary element $v$ in $T_{g} G$ is given by $\mathcal{G} v=\mathcal{K}^{-1}\left(v+T_{g} N^{\perp}\right)$. Writing $\mathcal{G}$ as $\mathcal{G}=\mathcal{F}+1$, it is straightforward to check that $\mathcal{F}$ satisfies $T_{g} N=\operatorname{Im}(\mathcal{F}+1)$ and

$$
\omega((\mathcal{F}+1) u,(\mathcal{F}+1) v)=G((\mathcal{F}+1) u,(\mathcal{F}-1) v)
$$

for arbitrary $u$ and $v$ in $T_{g} G$. Proceeding along the same lines as in subsection 3.3, one can see that the isometric character of $\mathcal{F}$ follows from the antisymmetric property of $\omega$.

Since $\left.\partial_{\tau} X\right|_{\partial \Sigma}$ in eq. (4.1) belongs to $T_{g} N$, it can be written as $\left.\partial_{\tau} X\right|_{\partial \Sigma}=(\mathcal{F}+1) v$ for some $v$ in $T_{g} G$. Upon noting (4.4), the boundary condition (4.1) takes the form

$$
G\left(\delta X,\left.\partial_{\sigma} X\right|_{\partial \Sigma}\right)=G(\delta X,(\mathcal{F}-1) v) \text { for all } \delta X \in T_{g} N
$$

This is equivalent to

$$
\left.\partial_{\sigma} X\right|_{\partial \Sigma}=(\mathcal{F}-1) v+v_{0},
$$

with arbitrary $v_{0}$ in $T_{g} N^{\perp}=\operatorname{Ker}(\mathcal{F}+1)$. Acting with $\mathcal{F}+1$ on both sides of this equation we finally have

$$
\left.(\mathcal{F}+1) \partial_{\sigma} X\right|_{\partial \Sigma}=(\mathcal{F}-1)(\mathcal{F}+1) v=\left.(\mathcal{F}-1) \partial_{\tau} X\right|_{\partial \Sigma}
$$

which is nothing but the gluing condition (2.12) written in terms of world sheet coordinates $\tau$ and $\sigma$.

Let us go back to eq. (4.3). If there exists $w^{\prime}-w \neq 0$ in $T_{g} N$ such that the equation holds and $z$ is taken equal to $w^{\prime}-w$, the right hand side of eq. (4.3) vanishes and it follows that $w^{\prime}-w$ is a null vector. Since Euclidean D-branes do not have null vectors, such a $w^{\prime}-w \neq 0$ does not exist and $\operatorname{det}\left(\left.G\right|_{N}-\omega\right) \neq 0$. The analysis of D-branes based on the gluing condition (2.8) then provides all Euclidean D-branes described by boundary conditions but may miss some Lorentzian or metrically degenerate D-branes for which $\operatorname{det}\left(\left.G\right|_{N}-\omega\right)=0$.

\section{An application: D-branes from global isometries}

From the analysis in subsection 3.2 it is convenient to distinguish two cases. The first one assumes that $F$ does not depend on $g$. We call such isometries constant or global and will be treated in this section. The second case accounts for $g$-dependent isometries $F(g)$. We call them local or nonconstant; some examples will be considered in section 5 .

If $F$ is a global (or constant) isometry solving involutivity, eq. (3.15) holds. Frobenius theorem ensures that $\Pi_{g}=g\left(\operatorname{Ad}_{g^{-1}} F-1\right) \mathfrak{g}$ is the tangent space to a submanifold $N$ of $G$ but it does not identify $N$. This problem we address next. 
Consider the vector field $t_{V}(g)=g\left(\operatorname{Ad}_{g^{-1}} F-1\right) V$, with $V$ in $\mathfrak{g}$, and let $g_{0}$ be a group element. By definition, the integral curve $\gamma_{t_{V}}\left(s ; g_{0}\right)$ of $t_{V}$ that goes through $g_{0}$ is the solution to

$$
\gamma_{t_{V}}\left(0 ; g_{0}\right)=g_{0} \quad \frac{d}{d s} \gamma_{t_{V}}\left(s ; g_{0}\right)=t_{V}\left(\gamma_{t_{V}}\left(s ; g_{0}\right)\right)
$$

where $s$ is a real parameter along the curve. Simple inspection shows that the solution is

$$
\gamma_{t_{V}}\left(s ; g_{0}\right)=e^{s F V} g_{0} e^{-s V} .
$$

The set $N_{g_{0}}$ of all points connected to $g_{0}$ by integral curves of vector fields $t_{V}$, with $V$ arbitrary, can always be written as

$$
N_{g_{0}}=\left\{e^{F V} g_{0} e^{-V}: V \in \mathfrak{g}\right\} .
$$

The only candidate for a D-brane containing $g_{0}$ is then $N_{g_{0}}$. According to section 3, however, the fact that $N_{g_{0}}$ contains the integral curves of all the fields $t_{V}$ that go through $g_{0}$ is not enough to conclude that $N_{g_{0}}$ is a D-brane. For this to be the case, $\Pi^{N_{g_{0}}}$ must be an involutive distribution. In summary, D-branes for constant $F$, if they exist, have the form of $N_{g_{0}}$ in (5.2).

\subsection{Automorphisms and twined conjugacy classes as D-branes}

Take $F=R$ with $R$ a Lie algebra automorphism compatible with condition (3.22). Automorphisms of this type form a group, denoted by $A u t_{\Omega}(\mathfrak{g})$. Being $R$ an automorphism, it satisfies

$$
R[U, V]=[R U, R V]
$$

for all $U$ and $V$ in $\mathfrak{g}$. For any such $F$, the involutivity equation (3.15) is solved by $W=[V, U]$ and the manifold $N_{g_{0}}$ is the $R$-twined conjugacy class $\mathcal{C}\left(R, g_{0}\right)$ of $g_{0}$,

$$
N_{g_{0}}=\mathcal{C}\left(R, g_{0}\right)=\left\{e^{R V} g_{0} e^{-V}: V \in \mathfrak{g}\right\} .
$$

In appendix $\mathrm{C}$ it is shown that the dimension of $\Pi_{g}=g\left(\operatorname{Ad}_{g^{-1}} F-1\right) \mathfrak{g}$ is constant for all $g$ in $\mathcal{C}\left(R, g_{0}\right)$. Furthermore, as proved at the end of section 3, the two-form $\omega$ given in (3.19) satisfies $\left.H\right|_{N_{g_{0}}}=d \omega$. Hence, $\mathcal{C}\left(R, g_{0}\right)$ is a D-brane [11, 13-15].

Note that for $R=1$, the manifold $N_{g_{0}}$ is a conventional conjugacy class $\mathcal{C}\left(1, g_{0}\right)$ [11]. Consider now $R \neq 1$ and assume that $R$ is an inner automorphism. By definition, it exists an $h$ in $G$ such that $R V=\operatorname{Ad}_{h} V$ for all $V$ in $\mathfrak{g}$. Since $\operatorname{Ad}_{h_{1}} \operatorname{Ad}_{h_{2}}=\operatorname{Ad}_{h_{1} h_{2}}$, inner automorphisms form a subgroup $\operatorname{Inn}_{\Omega}(\mathfrak{g})$. Automorphisms which are not inner are called outer and form the equivalence classes of the quotient $A u t_{\Omega}(\mathfrak{g}) / \operatorname{Inn}_{\Omega}(\mathfrak{g})$. Any automorphism $R$ can therefore be written as $R=R_{1} \tilde{R}_{2}$, with $R_{1}$ inner and $\tilde{R}_{2}$ of the same type as $R$. Consider the $\left(R_{1} \tilde{R}_{2}\right)$-twined conjugacy class of $g_{0}$. Using that $R_{1}=\operatorname{Ad}_{h}$ for some $h$ in $G$, and recalling that $e^{t \mathrm{Ad}_{r} U}=\operatorname{Ad}_{r} e^{t U}$ for all $r$ in $G$ and all $U$ in $\mathfrak{g}$, it follows that

$$
\mathcal{C}\left(R_{1} \tilde{R}_{2}, g_{0}\right)=h \mathcal{C}\left(\tilde{R}_{2}, h^{-1} g_{0}\right)
$$

for some $h$ in $G$. If $R$ is inner, so that $\tilde{R}_{2}=1$, the D-branes are the left translates by $h$ of the conventional conjugacy classes [13]. For $R$ outer, the D-branes are the translates of $\tilde{R}_{2}$-twined conjugacy classes $[13,22]$. 


\subsection{D-branes for semisimple Lie algebras}

Involutivity for isometries that are not Lie algebra automorphisms is more complicated. It has been suggested $[12,13,32]$ that isometries of the form $F=-R$, with $R$ a Lie algebra automorphism, define D-branes. In the sequel we investigate this issue and reach an answer in the negative.

Consider eq. (3.15) and make the change $2 Y=[U, V]-W$. Involutivity requires that, for all $U$ and $V$ in $\mathfrak{g}$ and for all $g$, there must exist $Y$ in $\mathfrak{g}$ such that

$$
g[U, V]=g\left(\operatorname{Ad}_{g^{-1}} R+1\right) Y .
$$

After multiplying from the left with $g^{-1}$, this becomes

$$
[\mathfrak{g}, \mathfrak{g}] \subset\left(\operatorname{Ad}_{g^{-1}} R+1\right) \mathfrak{g} .
$$

We restrict ourselves to semisimple Lie algebras $\mathfrak{g}$. Concerning this restriction, we make two comments. The first one is that if for the invariant metric $\Omega$ one takes a Killing form and $R$ is an arbitrary Lie algebra automorphism, $\pm R$ are isometries. ${ }^{2}$ The second observation is that two of the most relevant semisimple Lie algebras in string theory are $\mathfrak{s l}(2, \mathbf{R})$ and $\mathfrak{s u}(2)$, all whose isometries are either of the form $F=R$ or $F=-R$. As already mentioned, $F=R$ define D-branes. Consideration of $F=-R$ then completes the search of D-branes for constant isometries for $\mathfrak{s l}(2, \mathbf{R})$ and $\mathfrak{s u}(2)$.

Let $\mathfrak{g}$ be a semisimple Lie algebra of dimension $d$. Being semisimple, $[\mathfrak{g}, \mathfrak{g}]=\mathfrak{g}$ and the involutivity requirement (5.4) reads

$$
\mathfrak{g}=\left(\operatorname{Ad}_{g^{-1}} R+1\right) \mathfrak{g} .
$$

Let $D_{\mathrm{d}}$ denote the set of group elements where this condition holds. That is, $g$ belongs to $D_{\mathrm{d}}$ if $\left(\operatorname{Ad}_{g^{-1}} R+1\right) V \neq 0$ for all $V \neq 0$ in $\mathfrak{g}$. The tangent plane (3.5) at all $g$ in $D_{\mathrm{d}}$ is then $\Pi_{g}=T_{g} G$. The distribution $\Pi^{D_{\mathrm{d}}}$ is trivially involutive and is the tangent bundle to $D_{\mathrm{d}}$ itself. The only D-brane candidate provided by $F=-R$ is hence $D_{\mathrm{d}}$. According to our discussion in section 3 , for $D_{\mathrm{d}}$ to be a D-brane, the integral curves of the vector fields $t_{V}(g)=g\left(\operatorname{Ad}_{g^{-1}} R+1\right) V$ should be contained in $D_{\mathrm{d}}$. In the remaining of this section we show that this is not the case, thus implying that $F=-R$ does not define a D-brane.

The proof consists in (i) finding group elements $g$ outside $D_{\mathrm{d}}$, and (ii) showing that these $g$ are connected to elements in $D_{\mathrm{d}}$ by integral curves of the vector fields $t_{V}(g)$.

Proof of (i). A group element $g$ is not in $D_{\mathrm{d}}$ if there exists a nonzero $V$ in $\mathfrak{g}$ such that $\left(\operatorname{Ad}_{g^{-1}} R+1\right) V=0$. Let us call $D_{\mathrm{d}}^{-}$to the set formed by such $g$,

$$
D_{\mathrm{d}}^{-}=G-D_{\mathrm{d}}=\left\{g \in G: \operatorname{Ker}\left(\operatorname{Ad}_{g^{-1}} R+1\right) \neq 0\right\} .
$$

The group Aut $(\mathfrak{g})$ has in general several connected components, the component containing the identity being the normal subgroup $\operatorname{Inn}(\mathfrak{g})$, and the quotient $\operatorname{Aut}(\mathfrak{g}) / \operatorname{Inn}(\mathfrak{g})$ being a

\footnotetext{
${ }^{2}$ Note in this regard that a non-simple semisimple Lie algebra may have invariant metrics which are not proportional to its Killing form, in which case a Lie algebra automorphism need not be an isometry. The case of simple Lie algebras is more restrictive since all invariant metrics are proportional to the Killing form.
} 
finite group. It follows that in every component, and in particular in that containing $R$, there is then an automorphism $S$ such that $S^{n}=1$ for an integer $n$. This implies that the eigenvalues of $S$ can only be $n$-th roots of 1 . Furthermore, if a root $e^{-\mathrm{i} \theta}$ is an eigenvalue with multiplicity $m$, so is $e^{\mathrm{i} \theta}$. Since $S$ and $R$ are in the same component, they are related by an inner automorphism, meaning that there is an $h$ in $G$ such that $R=\operatorname{Ad}_{h} S$. We now distinguish three cases:

- $S$ has an eigenvalue -1 . For $g=h$, the operator $\operatorname{Ad}_{g^{-1}} R$ has then an eigenvalue -1 and $g$ is in $D_{\mathrm{d}}^{-}$.

- $S$ does not have an eigenvalue -1 but has two complex conjugate eigenvalues $e^{\mp \mathrm{i} \theta}$ with eigenvectors $Z_{1} \pm \mathrm{i} Z_{2}$,

$$
S\left(Z_{1} \pm \mathrm{i} Z_{2}\right)=e^{\mp \mathrm{i} \theta}\left(Z_{1} \pm \mathrm{i} Z_{2}\right) .
$$

Using that $\mathfrak{g}$ is semisimple and that $S$ is an automorphism, it is straightforward to show that $X=\left[Z_{1}, Z_{2}\right]$ is an eigenvector of $S$ with eigenvalue +1 and that there exists $a$ real such that $\left[X, Z_{1}\right]=a Z_{2}$ and $\left[X, Z_{2}\right]=-a Z_{1}$. The constant $a$ can be eliminated by redefining $Z_{1}, Z_{2}$ and $X$, so that

$$
\left[Z_{1}, Z_{2}\right]=X \quad\left[X, Z_{1}\right]=Z_{2} \quad\left[X, Z_{2}\right]=-Z_{1} .
$$

Take now $W=\frac{\pi}{\sqrt{2}}\left(Z_{1}-Z_{2}\right)$ and consider $g=h e^{-W}$. It follows after some algebra that the automorphism $\operatorname{Ad}_{g^{-1}} R=\operatorname{Ad}_{e^{W}} S$ has two eigenvectors with eigenvalue -1 ,

$$
\begin{aligned}
\operatorname{Ad}_{g^{-1}} R X & =-X \\
\operatorname{Ad}_{g^{-1}} R\left(\cos \theta Z_{1}+Z_{2}-\sin \theta Z_{2}\right) & =-\left(\cos \theta Z_{1}+Z_{2}-\sin \theta Z_{2}\right) .
\end{aligned}
$$

Hence $g=h e^{-W}$ belongs to $D_{\mathrm{d}}^{-}$.

- $S$ only has eigenvalues +1 . In this case, $S$ is the identity automorphism and $R$ is inner. For $\mathfrak{g}$ semisimple, it is always possible to take $X$ in its Cartan subalgebra and $Z_{1}$ and $Z_{2}$ in $\mathfrak{g}$ such that

$$
\left[Z_{1}, Z_{2}\right]=X \quad\left[X, Z_{1}\right]=Z_{1} \quad\left[X, Z_{2}\right]=-Z_{2} .
$$

It is then very simple to check that $\operatorname{Ad}_{g^{-1}} R$, where $g$ is taken as $g=h e^{-W}$ with $W=\sqrt{2} \pi\left(Z_{1}-Z_{2}\right)$, has two eigenvectors with eigenvalue -1 ,

$$
\begin{aligned}
\operatorname{Ad}_{g^{-1}} R X & =-X \\
\operatorname{Ad}_{g^{-1}} R\left(Z_{1}+Z_{2}\right) & =-\left(Z_{1}+Z_{2}\right) .
\end{aligned}
$$

So also in this case $D_{\mathrm{d}}^{-}$is not empty.

As shown in appendix $\mathrm{C}$, the spectrum of $\operatorname{Ad}_{g^{-1}} R$ is invariant under $R$-twined conjugation. Hence, if $g$ is in $D_{\mathrm{d}}^{-}$, the whole $R$-twined conjugacy class $\mathcal{C}(R, g)$ is in $D_{\mathrm{d}}^{-}$. As a result, $D_{\mathrm{d}}^{-}$is a union of $R$-twined conjugacy classes. It is clear that $D_{\mathrm{d}}^{-}$has dimension less than d. 
Proof of (ii). The tangent space $T_{g_{0}} G=g_{0} \mathfrak{g}$ at a $g_{0}$ in $D_{\mathrm{d}}^{-}$is most conveniently written as

$$
T_{g_{0}} G=g_{0}\left(\operatorname{Ad}_{g_{0}^{-1}} R-1\right) \mathfrak{g} \cup g_{0}\left(\operatorname{Ad}_{g_{0}^{-1}} R+1\right) \mathfrak{g} .
$$

Since the $R$-twined conjugacy class $\mathcal{C}\left(R, g_{0}\right)$ is contained in $D_{\mathrm{d}}^{-}$and the fields $g\left(\operatorname{Ad}_{g^{-1}} R-1\right) \mathfrak{g}$ generate motions inside $\mathcal{C}\left(R, g_{0}\right)$, there must be at least one vector field $t_{V}(g)=g\left(\operatorname{Ad}_{g^{-1}} R+1\right) V$ whose integral curve goes from $D_{\mathrm{d}}^{-}$to $D_{\mathrm{d}}$. Such a curve connects points in the D-brane with points outside the D-brane. We thus conclude that $D_{\mathrm{d}}$ cannot be a D-brane.

This proves that there are no D-branes for a semisimple Lie algebra $\mathfrak{g}$ and $F=-R$, with $R$ a constant automorphism. This result contrasts with previous studies on the subject [12]. If the requirement that $D_{\mathrm{d}}$ contain the integral curves of all the fields $t_{V}$ were relaxed, $D_{\mathrm{d}}$ would be a D-brane of dimension d, provided it exists a suitable two-form $\omega$. This D-brane would not be filling, since $D_{\mathrm{d}}^{-}$is not empty. Furthermore, it would exclude allowed motions for the string endpoints, thus contradicting the definition of D-brane.

\section{Some considerations on D-branes for local isometries}

For local isometries $F(g)$, involutivity takes the form (3.14). Given a local isometry $F(g)$, it is always possible to construct a new isometry

$$
F(g) \rightarrow F^{\prime}(g)=\operatorname{Ad}_{g} F^{-1}(g) \operatorname{Ad}_{g}
$$

It is very easy to convince oneself that, at any point $g$ in $G$, both $F$ and $F^{\prime}$ define the same tangent space $\Pi_{g}=g\left(\operatorname{Ad}_{g^{-1}} F-1\right) \mathfrak{g}$. They thus define the same distribution. Furthermore, it is straightforward to check that $F^{\prime}$ satisfies the involutivity condition (3.14) if and only if $F$ does. Assume that this is the case, so that they define the same submanifold $N$ of $G$.

The gluing conditions (2.8) for $F$ and $F^{\prime}$ read

$$
\begin{gathered}
F:\left.\quad(\mathcal{F}-1) \partial_{\tau} X\right|_{\partial \Sigma}=\left.(\mathcal{F}+1) \partial_{\sigma} X\right|_{\partial \Sigma} \\
F^{\prime}:\left.\quad\left(\mathcal{F}^{\prime}-1\right) \partial_{\tau} X\right|_{\partial \Sigma}=\left.\left(\mathcal{F}^{\prime}+1\right) \partial_{\sigma} X\right|_{\partial \Sigma}
\end{gathered}
$$

where the matrices $\mathcal{F}$ and $\mathcal{F}^{\prime}$ are given by $\mathcal{F}=-\bar{e}^{-1} F e$ and $\mathcal{F}^{\prime}=-\bar{e}^{-1} F^{\prime} e$. Noting that $\mathcal{F}^{\prime}=\mathcal{F}^{-1}$, eq. (6.3) can be written, after multiplication from the left with $\mathcal{F}$, as

$$
F^{\prime}: \quad-\left.(\mathcal{F}-1) \partial_{\tau} X\right|_{\partial \Sigma}=\left.(\mathcal{F}+1) \partial_{\sigma} X\right|_{\partial \Sigma}
$$

The gluing condition (6.4) for $F^{\prime}$ has a relative negative sign as compared to the gluing condition (6.2) for $F$. This sign has important consequences for the recasting of the corresponding gluing conditions as boundary conditions. Indeed, the two-forms $\omega$ and $\omega^{\prime}$ associated to $F$ and $F^{\prime}$ are related by $\omega^{\prime}=-\omega$, so the conditions $d \omega=\left.H\right|_{N}$ and $d \omega^{\prime}=\left.H\right|_{N}$ cannot generally hold simultaneously. Let us see an example. 
Example: Filling D-brane. In this case, the sigma model boundary conditions (2.1) become

$$
\left.\omega_{\mu \nu} \partial_{\tau} X^{\nu}\right|_{\partial \Sigma}=\left.G_{\mu \nu} \partial_{\sigma} X^{\nu}\right|_{\partial \Sigma} .
$$

Assume that the D-brane is defined by an isometry $F$. This requires in particular that the gluing condition (6.2) can be written as in (6.5), with $\omega$ such that $d \omega=H$. See ref. [21] for some examples. The gluing condition (6.4) can then be written in the form (6.5), but needs $\omega^{\prime}=-\omega$, and $d \omega^{\prime} \neq H$. The isometry $F^{\prime}$ hence does not define a D-brane.

We close this section by further illustrating that an integrable gluing condition by itself does not define a D-brane. Consider $F(g)=-\operatorname{Ad}_{g}$. The tangent plane (3.5) at all $g$ in $G$ is $\Pi_{g}=T_{g} G$. The isometry $F$ defines trivially an involutive distribution, the submanifold $N$ being the whole group $G$. Since $\mathcal{F}=1$, the gluing condition (6.2) becomes $\left.\partial_{\sigma} X^{\mu}\right|_{\partial \Sigma}=0$. This, in turn, cannot be understood as a sigma model boundary condition, since it requires $\omega=0$ on the whole group manifold and does not account for a nontrivial $H$.

\section{Conclusion}

Given a WZW model with real Lie group $G$, Lie algebra $\mathfrak{g}$ and invariant Lie algebra metric $\Omega$, we have shown that a linear map $F(g)$ acting on $\mathfrak{g}$ defines a D-brane if the following conditions hold:

(i) $F(g)$ is an isometry of $\Omega$.

(ii) The vector fields $t_{A}=F T_{A} g-g T_{A}=t^{\mu}{ }_{A} \partial_{\mu}$ defined by $F(g)$ span a distribution. That is, the matrix formed by the coefficients $t^{\mu}{ }_{A}$ has constant rank on a submanifold $N$ of the group manifold. If this is the case and the rank is $p+1$, there are $p+1$ linearly independent vector fields $k_{i}$ that are linear combinations $k_{i}=c_{i A} t_{A}$ of the fields $t_{A}$.

(iii) The integral curves of the fields $k_{i}$ are contained in $N$.

(iv) The fields $k_{i}$ are involutive in $N$.

(v) The two-form $\omega$ globally defined on $N$ by its action $\omega\left(k_{i}, k_{j}\right)$ on the fields $k_{i}=c_{i A} t_{A}$ through $\omega\left(t_{A}, t_{B}\right)=\Omega\left(\operatorname{Ad}_{g^{-1}} F T_{A}-T_{A}, \operatorname{Ad}_{g^{-1}} F T_{B}+T_{B}\right)$ satisfies $d \omega=\left.H\right|_{N}$.

The conditions above account for both metrically nondegenerate and degenerate D-branes. They are met by $F$ any constant $\Omega$-preserving Lie algebra automorphism $R$, so the well known result [11-14] that the $R$-twined conjugacy classes of the group $G$ are D-branes extends to metrically degenerate classes.

WZW models based on semisimple Lie algebras are of particular interest in string theory, two of the most studied models being $\mathfrak{s u}(2)$ and $\mathfrak{s l}(2, \mathbf{R})$. It had been claimed that constant $F=-R$ could provide D-branes for such models. This has been disproved in this paper, since condition (iii) above fails.

For more general scenarios, (ii)-(v) must be checked for any given isometry $F$. This is however straightforward. In ref. [21] the Nappi-Witten model [34] is considered and 
several families of D-branes for $g$-dependent isometries $F(g)$ are found, some have Euclidean signature, some have Lorentzian and some are metrically degenerate. It would be interesting to study if D-branes defined by $g$-dependent isometries have a translate in the algebraic framework, since normal ordering ambiguities may occur. Our interest in this paper has been the geometric description of D-branes in WZW string backgrounds taking as starting point a gluing condition $J_{+}=F J_{-}$that matches the chiral currents at the world sheet boundary. It remains an open problem to study if the geometric approach presented here describes D-branes for which a full set of gluing conditions have not been found, the so-called permutation D-branes [19] among them.

\section{Acknowledgments}

The authors are grateful to C. Moreno for conversations, and to MEC and UCM-BSCH, Spain for partial support through grants FPA2008-04906 and 910770-GR35/10-A.

\section{A Alternative derivation of eq. (3.2)}

Here we present an alternative derivation of eq. (3.2). The idea is to solve the gluing condition (2.12) for $\partial_{\tau} x^{\mu}$ in terms of the eigenvectors of the matrix $\mathcal{F}$.

The (generalized) eigenvectors of the matrix $\mathcal{F}$ form a basis of linearly independent vectors. An eigenvalue $\lambda$ with algebraic multiplicity $a_{\lambda}$ and geometric multiplicity $m_{\lambda}$ has $i=1, \ldots, m_{\lambda}$ eigenvectors $v_{(\lambda, i, 1)}$ and $a_{\lambda}-m_{\lambda}$ generalized eigenvectors that can be organized in $m_{\lambda}$ chains

$$
(\mathcal{F}-\lambda) v_{(\lambda, i, 1)}=0 \quad \ldots \quad(\mathcal{F}-\lambda) v_{\left(\lambda, i, \ell_{i}\right)}=v_{\left(\lambda, i, \ell_{i}-1\right)} \quad \ell_{i}=2, \ldots, L_{i} .
$$

The index $\ell_{i}=1, \ldots, L_{i}$ labels the members of the chain $(\lambda, i)$. Every chain is headed by an eigenvector $v_{(\lambda, i, 1)}$ and terminates in a highest- $\ell_{i}$ generalized eigenvector $v_{\lambda, i, L_{i}}$. Consider two arbitrary (generalized) eigenvectors $v_{\left(\lambda, i, \ell_{i}\right)}$ and $v_{\left(\mu, j, m_{j}\right)}$ relative to the eigenvalues $\lambda$ and $\mu$.

Since the (generalized) eigenvectors $\left\{v_{\left(\lambda, i, \ell_{i}\right)}\right\}$ are linearly independent, $\left.\partial_{\tau} X\right|_{\partial \Sigma}$ and $\left.\partial_{\sigma} X\right|_{\partial \Sigma}$ are linear combinations

$$
\left.\partial_{\tau} X\right|_{\partial \Sigma}=\left.\sum_{\lambda, i, \ell_{i}} \alpha_{\left(\lambda, i, \ell_{i}\right)} v_{\left(\lambda, i, \ell_{i}\right)} \quad \partial_{\sigma} X\right|_{\partial \Sigma}=\sum_{\lambda, i, \ell_{i}} \beta_{\left(\lambda, i, \ell_{i}\right)} v_{\left(\lambda, i, \ell_{i}\right)}
$$

with coefficients $\alpha_{\left(\lambda, i, \ell_{i}\right)}$ and $\beta_{\left(\lambda, i, \ell_{i}\right)}$. Upon substitution in eq. (2.12), the following set of equations follows for every chain $(\lambda, i)$

$$
\begin{aligned}
\alpha_{\left(\lambda, i, \ell_{i}\right)}+(\lambda-1) \alpha_{\left(\lambda, i, \ell_{i}-1\right)} & =\beta_{\left(\lambda, i, \ell_{i}\right)}+(\lambda+1) \beta_{\left(\lambda, i, \ell_{i}-1\right)} \quad \ell_{i}=2, \ldots, L_{i} \\
(\lambda-1) \alpha_{\left(\lambda, i, L_{i}\right)} & =(\lambda+1) \beta_{\left(\lambda, i, L_{i}\right)} .
\end{aligned}
$$

We must solve eqs. (A.2)-(A.3) for $\alpha_{\left(\lambda, i, \ell_{i}\right)}$ in terms of $\beta_{\left(\lambda, i, \ell_{i}\right)}$. To this end, we consider the cases $\lambda=-1, \lambda=1$ and $\lambda \neq \pm 1$ separately. 
- Assume that $\mathcal{F}$ has a chain $\left\{v_{\left(-1, i, \ell_{i}\right)}\right\}$ relative to the eigenvalue $\lambda=-1$. Eq. (A.3) implies $\alpha_{\left(-1, i, L_{i}\right)}=0$, so the vector $v_{\left(-1, i, L_{i}\right)}$ does not occur in $\partial_{\tau} x$. Eq. (A.2) in turn implies that there are infinitely many solutions for $\alpha_{(-1, i, 1)} \ldots \alpha_{\left(-1, i, L_{i}-1\right)}$; one for every choice of $\beta_{(-1, i, 1)} \ldots \beta_{\left(-1, i, L_{i},\right)}$. The (generalized) eigenvectors $v_{(-1, i, 1)} \cdots v_{\left(-1, i, L_{i}-1\right)}$ then occur in $\partial_{\tau} x$.

- Look next at a chain $\left\{v_{\left(1, i, \ell_{i}\right)}\right\}$ with eigenvalue $\lambda=1$. Eq. (A.3) now requires $\beta_{\left(1, i, L_{i}\right)}=0$ and leaves $\alpha_{\left(1, i, L_{i}\right)}$ arbitrary. This and eq. (A.2) give arbitrary solutions for all $\alpha_{\left(1, i, \ell_{i}\right)}$. In this case, all the vectors in the chain are tangent.

- Consider finally a chain $\left\{v_{\left(\lambda, i, \ell_{i}\right)}\right\}$ relative to an eigenvalue $\lambda \neq \pm 1$. Eqs. (A.2)-(A.3) give arbitrary solutions for all $a_{\left(\lambda, i, \ell_{i}\right)}$ and again all the vectors in the chain occur in $\partial_{\tau} x$.

The space $\Pi_{g}$ of tangent directions is then

$$
\Pi_{g}=\operatorname{Span}\left\{v_{\left(\lambda, i, \ell_{i}\right)}:\left(\lambda, \ell_{i}\right) \neq\left(-1, L_{i}\right)\right\}
$$

and has dimension $\mathrm{d}-m_{-1}$, where we recall that $\mathrm{d}$ is the group dimension and $m_{-1}$ the geometric multiplicity of $\lambda=-1$. Since the nontangent vectors $v_{\left(-1, i, L_{i}\right)}$ are removed from the set of all (generalized) eigenvectors through the action of $\mathcal{F}+1$, one has

$$
\Pi_{g}=(\mathcal{F}+1) \operatorname{Span}\left\{v_{\left(\lambda, i, \ell_{i}\right)}\right\}=\operatorname{Im}(\mathcal{F}+1) .
$$

This is precisely eq. (3.2).

\section{B Metrically degenerate tangent planes}

Here we explicitely construct tangent vectors that are orthogonal to all tangent vectors, including itself, so they define a metrically degenerate tangent plane $\Pi_{g}$.

To this end, we first note that the isometry property (3.20) and eq. (A.1) imply the orthogonality relation

$$
(1-\lambda \mu) G\left(v_{\left(\lambda, i, \ell_{i}\right)}, v_{\left(\mu, j, m_{j}\right)}\right)=0 .
$$

for two arbitrary (generalized) eigenvectors.

Assume for concreteness that there is only one chain $\left\{v_{-1,1,1} \ldots v_{(-1,1, L)}\right\}$ of $L \geq 2$ generalized eigenvectors relative to the eigenvalue $\lambda=-1$, and let us write $u_{\ell}:=v_{(-1,1, \ell)}$ for its members. As explained in appendix A, the first $L-1$ vectors in this chain define directions in $\Pi_{g}$. Noting that $\mathcal{F}$ is an isometry and recalling eqs. (A.1), we have

$$
G\left(u_{1}, u_{\ell+1}\right)=G\left(\mathcal{F} u_{1}, \mathcal{F} u_{\ell+1}\right)=G\left(u_{1}, u_{\ell+1}\right)-G\left(u_{1}, u_{\ell}\right) \quad \ell=1, \ldots, L-1 .
$$

It follows that $G\left(u_{1}, u_{\ell}\right)=0$ for $\ell=1 \ldots, L-1$. Since $\left\{u_{\ell}\right\}$ is the only chain with eigenvalue -1 , any other direction in $\Pi_{g}$ has eigenvalue $\lambda \neq-1$, and thus eq. (B.1) implies that it is orthogonal to $u_{1}$. The eigenvector $u_{1}$ is thus orthogonal to all (generalized) eigenvectors spanning $\Pi_{g}$, and in particular to itself.

It is trivial to extend these arguments to show that every eigenvector heading a chain with eigenvalue $\lambda=-1$ defines a null direction orthogonal to $\Pi_{g}$. 


\section{Invariance of the spectrum of $\mathrm{Ad}_{g^{-1}} R$}

This appendix contains the discussion of the invariance of the spectrum of the operator $\operatorname{Ad}_{g^{-1}} R$ under $R$-twined conjugation, where $R$ is a Lie algebra automorphism.

The eigenvalue problem for $\operatorname{Ad}_{g^{-1}} R$ takes the form

$$
R V_{(\lambda, \ell)} g=\lambda g V_{(\lambda, \ell)}+g V_{(\lambda, \ell-1)},
$$

where the last term accounts for the occurrence of generalized eigenvectors. Here the chain labeling index $i$ in appendices $\mathrm{A}$ and $\mathrm{B}$ has been omitted in the notation since it does not play any rôle. An arbitrary $R$-twined conjugate $g^{\prime}$ of $g$ can be written as

$$
g^{\prime}=e^{-R U} g e^{U},
$$

for some $U$ in $\mathfrak{g}$. After some trivial manipulations, eq. (C.1) can be written in terms of $g^{\prime}$ as

$$
e^{-R U} R V_{(\lambda, \ell)} e^{R U} g^{\prime}=\lambda g^{\prime} e^{-U} V_{(\lambda, \ell)} e^{U}+g^{\prime} e^{-U} V_{(\lambda, \ell-1)} e^{U} .
$$

Being $R$ a Lie algebra automorphism, the left hand side of this equation is $R\left(e^{-U} V_{(\lambda, \ell)} e^{U}\right) g^{\prime}$. Eq. (C.2) becomes then

$$
\operatorname{Ad}_{g^{\prime-1}} R V_{(\lambda, \ell)}^{\prime}=\lambda V_{(\lambda, \ell)}^{\prime}+V_{(\lambda, \ell-1)}^{\prime} \quad V_{(\lambda, \ell)}^{\prime}=e^{-U} V_{(\lambda, \ell-1)} e^{U} .
$$

The eigenvalues thus remain invariant, while the (generalized) eigenvectors change by ordinary conjugation. As a consequence, the dimension of the linear space generated by the eigenvectors associated to a given eigenvalue is constant under $R$-twined conjugation.

\section{References}

[1] E. Witten, Bound states of strings and p-branes, Nucl. Phys. B 460 (1996) 335 [hep-th/9510135] [SPIRES].

[2] C.-S. Chu and P.-M. Ho, Noncommutative open string and D-brane, Nucl. Phys. B 550 (1999) 151 [hep-th/9812219] [SPIRES].

[3] V. Schomerus, D-branes and deformation quantization, JHEP 06 (1999) 030 [hep-th/9903205] [SPIRES].

[4] N. Seiberg and E. Witten, String theory and noncommutative geometry, JHEP 09 (1999) 032 [hep-th/9908142] [SPIRES].

[5] C.-S. Chu and P.-M. Ho, Noncommutative D-brane and open string in pp-wave background with B-field, Nucl. Phys. B 636 (2002) 141 [hep-th/0203186] [SPIRES].

[6] A.Y. Alekseev, A. Recknagel and V. Schomerus, Non-commutative world-volume geometries: branes on $\mathrm{SU}(2)$ and fuzzy spheres, JHEP 09 (1999) 023 [hep-th/9908040] [SPIRES].

[7] G. Horcajada and F. Ruiz Ruiz, Quantization of the open string on plane-wave limits of $d S_{n} \times S^{n}$ and non-commutativity outside branes, Nucl. Phys. B 799 (2008) 110 [arXiv:0711.2991] [SPIRES].

[8] J. Rahmfeld and A. Rajaraman, The GS string action on $A d S_{3} \times S^{3}$ with Ramond-Ramond charge, Phys. Rev. D 60 (1999) 064014 [hep-th/9809164] [SPIRES]. 
[9] N. Berkovits, C. Vafa and E. Witten, Conformal field theory of AdS background with Ramond-Ramond flux, JHEP 03 (1999) 018 [hep-th/9902098] [SPIRES].

[10] N. Berkovits, M. Bershadsky, T. Hauer, S. Zhukov and B. Zwiebach, Superstring theory on $A d S_{2} \times S^{2}$ as a coset supermanifold, Nucl. Phys. B 567 (2000) 61 [hep-th/9907200] [SPIRES].

[11] A.Y. Alekseev and V. Schomerus, D-branes in the WZW model, Phys. Rev. D 60 (1999) 061901 [hep-th/9812193] [SPIRES].

[12] S. Stanciu, D-branes in an AdS 3 background, JHEP 09 (1999) 028 [hep-th/9901122] [SPIRES].

[13] S. Stanciu, D-branes in group manifolds, JHEP 01 (2000) 025 [hep-th/9909163] [SPIRES].

[14] S. Stanciu, A note on D-branes in group manifolds: flux quantization and D0-charge, JHEP 10 (2000) 015 [hep-th/0006145] [SPIRES].

[15] J.M. Figueroa-O'Farrill and S. Stanciu, More D-branes in the Nappi-Witten background, JHEP 01 (2000) 024 [hep-th/9909164] [SPIRES].

[16] C. Bachas and M. Petropoulos, Anti-de-Sitter D-branes, JHEP 02 (2001) 025 [hep-th/0012234] [SPIRES].

[17] S. Ribault and V. Schomerus, Branes in the 2D black hole, JHEP 02 (2004) 019 [hep-th/0310024] [SPIRES].

[18] Y. Hikida, R.R. Nayak and K.L. Panigrahi, D-branes in a big bang/big crunch universe: Nappi-Witten gauged WZW model, JHEP 05 (2005) 018 [hep-th/0503148] [SPIRES].

[19] S. Fredenhagen and T. Quella, Generalised permutation branes, JHEP 11 (2005) 004 [hep-th/0509153] [SPIRES].

[20] Y.-K.E. Cheung and L. Freidel, Inner brane: a D3-brane in the Nappi-Witten model from an inner group automorphism, Phys. Rev. D 79 (2009) 126007 [arXiv:0905.0540] [SPIRES].

[21] R. Hernández, G. Horcajada and F.R. Ruiz, D-branes with Lorentzian signature in the Nappi-Witten model, JHEP 08 (2011) 047 [arXiv: 1104.4730] [SPIRES].

$[22]$ L. Birke, J. Fuchs and C. Schweigert, Symmetry breaking boundary conditions and WZW orbifolds, Adv. Theor. Math. Phys. 3 (1999) 671 [hep-th/9905038] [SPIRES].

[23] J. Fuchs and C. Schweigert, Symmetry breaking boundaries. I: general theory, Nucl. Phys. B 558 (1999) 419 [hep-th/9902132] [SPIRES].

[24] G. Felder, J. Fröhlich, J. Fuchs and C. Schweigert, The geometry of WZW branes, J. Geom. Phys. 34 (2000) 162 [hep-th/9909030] [SPIRES].

[25] V. Schomerus and H. Saleur, The GL(1|1) WZW model: from supergeometry to logarithmic CFT, Nucl. Phys. B 734 (2006) 221 [hep-th/0510032] [SPIRES].

[26] G. Götz, T. Quella and V. Schomerus, The WZNW model on PSU $(1,1 \mid 2)$, JHEP 03 (2007) 003 [hep-th/0610070] [SPIRES].

[27] H. Saleur and V. Schomerus, On the $\mathrm{SU}(2 \mid 1)$ WZNW model and its statistical mechanics applications, Nucl. Phys. B 775 (2007) 312 [hep-th/0611147] [SPIRES].

[28] T. Quella, V. Schomerus and T. Creutzig, Boundary spectra in superspace $\sigma$-models, JHEP 10 (2008) 024 [arXiv:0712.3549] [SPIRES]. 
[29] T. Creutzig and V. Schomerus, Boundary correlators in supergroup WZNW models, Nucl. Phys. B 807 (2009) 471 [arXiv:0804.3469] [SPIRES].

[30] E. Witten, Nonabelian bosonization in two dimensions, Commun. Math. Phys. 92 (1984) 455 [SPIRES].

[31] C. Klimčík and P. Ševera, Open strings and D-branes in WZNW models, Nucl. Phys. B 488 (1997) 653 [hep-th/9609112] [SPIRES].

[32] S. Stanciu and A.A. Tseytlin, D-branes in curved spacetime: Nappi-Witten background, JHEP 06 (1998) 010 [hep-th/9805006] [SPIRES].

[33] W.M. Boothby, An introduction to differentiable manifolds and Riemannian geometry, Academic Press, New York U.S.A. (2003).

[34] C.R. Nappi and E. Witten, A WZW model based on a nonsemisimple group, Phys. Rev. Lett. 71 (1993) 3751 [hep-th/9310112] [SPIRES].

[35] T. Quella and V. Schomerus, Symmetry breaking boundary states and defect lines, JHEP 06 (2002) 028 [hep-th/0203161] [SPIRES].

[36] T. Quella, On the hierarchy of symmetry breaking D-branes in group manifolds, JHEP 12 (2002) 009 [hep-th/0209157] [SPIRES].

[37] J.M. Maldacena, G.W. Moore and N. Seiberg, Geometrical interpretation of D-branes in gauged WZW models, JHEP 07 (2001) 046 [hep-th/0105038] [SPIRES]. 